Pacific Journal of Mathematics

IA VAN RAPPEN THEOREm 


\section{ON THE VAN KAMPEN THEOREM}

\section{RiChaRd H. CROWELl}

1. Introduction. The van Kampen theorem provides a defining set of generators and relations for the fundamental group of the union of two topological spaces $X$ and $\mathrm{Y}$ where the fundamental groups of $X$, $Y$, and their intersection are given by defining sets of generators and relations. An intrinsic, purely group-theoretic formulation has been given by Fox using his direct limits of systems of groups [4]; however, the corresponding abstract proof had not been worked out. The present paper supplies such a proof (distilled from an earlier proof by Fox of the van Kampen theorem) to a natural generalization of the van Kampen theorem, which includes for example, in addition to the original theorem, the determination of the fundamental group of the union of an increasing nest of open sets each of whose groups is known [2].

In proving the principal result, Theorem (3.1), we depart from the usual development of the fundamental group in that paths and loops are not required to have the fixed unit interval as domains. In particular, a path $a$ is a continuous mapping of the interval $[0,\|a\|]$ into the space in question for some $\|a\| \geq 0$. For paths $a:[0,\|a\|] \rightarrow X$ and $b:[0,\|b\|] \rightarrow X$ which satisfy $a(\|a\|)=b(0)$, we define the product path $a \cdot b$ by

$$
a \cdot b(t)=\left\{\begin{array}{l}
a(t) \text { for } 0 \leq t \leq\|a\| \\
b(t-\|a\|) \text { for }\|a\| \leq t \leq\|a\|+\|b\| .
\end{array}\right.
$$

Thus, path multiplication is associative. Paths $a$ and $b$, having the same initial and terminal points, are equivalent, denoted by $a \simeq b$, iff there exists a collection of paths $h_{s}:\left[0,\left\|h_{s}\right\|\right] \rightarrow X, 0 \leq s \leq 1$, such that

$$
\begin{aligned}
& h_{0}=a \text { and } h_{1}=b, \\
& h_{s}(0)=a(0)=b(0), \\
& h_{s}\left(\left\|h_{s}\right\|\right)=a(\|a\|)=b(\|b\|), \\
& \left\|h_{s}\right\| \text { is a continuous function of } s, \\
& h_{s}(t) \text { is simultaneously continuous in } s \text { and } t .
\end{aligned}
$$

We note that, for any path $a$ and positive number $t$, there is a path $b$ equivalent to $a$ with $\|b\|=t$. Furthermore, $\left\|h_{s}\right\|$ can always be taken as a linear function of $s$ and thus, in view of the preceding sentence, may be arranged to be constant. The induced multiplication of equivalence classes of paths and the definitions of the fundamental groupoid and group of $X$ are made in the usual way.

Received July 8, 1958. 
2. Systems of groups and direct limits (cf. [4]). A system is any collection $\subseteq$ of groups and homomorphisms such that if $\theta ; G_{\alpha} \rightarrow G_{\beta}$ is in $\mathfrak{S}$, then $G_{\alpha}$ and $G_{\beta}$ are in $\mathfrak{S}$. A homomorphism $\Phi ; \mathfrak{S} \rightarrow G$ of $a$ system $\subseteq$ into a group $G$ is a function which assigns to each group $G_{\alpha}$ in $\subseteq$ a homomorphism $\varphi_{\alpha}: G_{\alpha} \rightarrow G$ such that, for every $\theta: G_{\alpha} \rightarrow G_{\beta}$ in $\mathfrak{S}$, we have $\varphi_{\alpha}=\varphi_{\beta} \theta$. The image of $\Phi$ is the smallest subgroup of $G$ which contains the image of every homomorphism $\varphi_{\alpha}$ in $\Phi$, and $\Phi$ is onto iff its image is $G$ itself.

A homomorphism $\Phi: \subseteq \rightarrow G$ is a direct limit iff (i) $\Phi$ is onto and (ii) for any group $H$ and homomorphism $\Psi: \subseteq \rightarrow H$, there exists a homomorphism $\lambda: G \rightarrow H$ such that $\Psi=\lambda \Phi$, that is, for every group $G_{\infty}$ in $\mathfrak{S}, \psi_{a}=\lambda \varphi_{a}$.

(2.1) THEOREM Any system $\subseteq$ has a direct limit unique to within isomorphism.

The proof is straightforward and is given in [4]. As a result of (2.1), one may relax the above terminology and speak simply of the group $G$ as the direct limit of the system $\mathfrak{S}$.

A given system $\mathfrak{S}$ may always be enlarged to a system $\mathfrak{S}^{\prime}$ by adjoining all, or any number of, identity homomorphisms and finite compositions of homomorphisms of $\mathfrak{S}$. It is obvious that any homomorphism of $\mathfrak{S}$ is also a homomorphism of $\mathfrak{S}^{\prime}$, and conversely. Thus,

(2.2) Any direct limit $\Phi: \subseteq \rightarrow G$ is a direct limit $\Phi: \mathfrak{S}^{\prime} \rightarrow G$, and conversely.

3. The generalized van Kampen theorem. Consider a collection of pathwise-connected, open subsets $X_{\alpha}$ of a topological space $X$ closed under finite intersections and such that

$$
\begin{aligned}
& X=\bigcup X_{\alpha} \\
& p \in \bigcap X_{\alpha}, \text { for some point } p
\end{aligned}
$$

The set $\mathfrak{S}$ of fundamental groups $G_{\alpha}=\pi\left(X_{\alpha}, p\right)$ and all homomorphisms $\theta: G_{\alpha} \rightarrow G_{\beta}$ induced by inclusion is a system, and the homomorphisms $\varphi_{\alpha}: G_{\alpha} \rightarrow G=\pi(X, p)$ induced by inclusion constitute a homomorphism $\Phi: \subseteq \rightarrow G$.

(3.1) VAN KAMPEN TheOREM. $\Phi: \subseteq \rightarrow G$ is a direct limit.

Proof. There are two propositions to verify :

I. $\Phi$ is onto. Consider an arbitrary non-trivial element $A \in G$ and a loop $a$ representing $A$. Since $A \neq 1$, we know that $\|a\|>0$. We construct a subdivision. 


$$
0=t_{0}<t_{1}<\cdots<t_{n}=\|a\|
$$

such that each $t_{i}-t_{i-1}$ is less than the Lebesgue number of the open covering of $[0,\|a\|]$ consisting of all inverse images $a^{-1} X_{x}$. We then choose $X_{\alpha_{i}}, i=1, \cdots, n$, such that

$$
a\left[t_{i-1}, t_{i}\right] \subset X_{\alpha_{i}}
$$$$
i=1, \cdots, n \text {. }
$$

For each point $t_{i}, i=0, \cdots, n$, of the subdivision, we select a path $b_{i}$ in $X$ subject to the conditions :

(i ) $b_{i}(0)=p$ and $b_{i}\left(\left\|b_{i}\right\|\right)=a\left(t_{i}\right)$

(ii) If $a\left(t_{i}\right)=p$, then $b_{i} \equiv p$

(iii) $b_{i}(t) \in X_{\alpha_{i}} \cap X_{\alpha_{i+1}}, \quad 0 \leq t \leq\left\|b_{i}\right\|$ and $i=1, \cdots, n-1$.

Note that (iii) uses the fact that the collection of subsets $X_{\alpha}$ is closed under finite intersections. Next, consider paths $a_{i}:\left[0, t_{i}-t_{i-1}\right] \rightarrow X, i=$ $1, \cdots, n$, defined by $a_{i}(t)=a\left(t+t_{i-1}\right)$.

Clearly,

$$
a=\prod_{i=1}^{n} a_{i}
$$

and

$$
a \simeq \prod_{i=1}^{n} b_{i-1} \cdot a_{i} \cdot b_{i}^{-1} .
$$

Each path $b_{i-1} \cdot a_{i} \cdot b_{i}^{-1}$ is a $p$-based loop whose image lies entirely in $X_{\alpha_{i}}$ and which, therefore, is a representative loop of $\varphi_{\alpha_{i}} A_{i}$ for some $A_{i} \in G_{\alpha_{i}}$. Thus,

$$
A=\prod_{i=1}^{n} \varphi_{\alpha_{i}} A_{i}
$$

and the proof of $\mathrm{I}$ is complete.

II. For any group $H$ and homomorphism $\Psi: \mathfrak{S} \rightarrow H$, there exists a homomorphism $\lambda: G \rightarrow H$ such that $\Psi=\lambda \Phi$.

Proving II obviously amounts to proving that, for any $A_{i} \in G_{\alpha_{i}}, i=$ $1, \cdots, r$,

$$
\prod_{i=1}^{r} \varphi_{a_{i}} A_{i}=1 \text { implies } \prod_{i=1}^{r} \psi_{\alpha_{i}} A_{i}=1 .
$$

We select representative loops $a_{i} \in A_{i}, i=1, \cdots, r$. Then the product

$$
a=\prod_{i=1}^{r} \varphi_{a_{i}} a_{i}
$$

is contractible (We denote an inclusion mapping and its induced homo- 
morphism of the fundamental groups by the same symbol), and there exists a homotopy $h: R \rightarrow X$, where $R=[0,\|a\|] \times[0,1]$, which satisfies

$$
\begin{aligned}
& h(t, 0)=a(t) \\
& h(0, s)=h(t, 1)=h(\|a\|, s)=p
\end{aligned}
$$

The vertical lines $t=\sum_{k=1}^{i}\left\|a_{k}\right\|, i=1, \cdots, r$, provide a decomposition of $R$, and we consider a refinement

$$
\begin{aligned}
& 0=t_{0}<t_{1}<\cdots<t_{n}=\|a\| \\
& 0=s_{0}<s_{1}<\cdots<s_{m}=1
\end{aligned}
$$

into rectangles

$$
R_{i j}=\left\{(t, s) \mid t_{i-1} \leq t \leq t_{i} \text { and } s_{j-1} \leq s \leq s_{j}\right\}
$$

the maximum of whose diameters is less than the Lebesgue number of the open covering of $R$ consisting of all inverse images $h^{-1} X_{\alpha}$. Consequently, there exists a function $\alpha(i, j)$ such that

$$
h\left(R_{i j}\right) \subset X_{\alpha(i, j)} \quad i=1, \cdots, n \text { and } j=1, \cdots, m .
$$

For each lattice point $\left(t_{i}, s_{j}\right)$, we select a path $e_{i j}$ in $X$ subject to the following conditions.

(iv) The initial and terminal points of $e_{i j}$ are $p$ and $h\left(t_{i}, s_{j}\right)$, respectively.

( v ) If $h\left(t_{i}, s_{j}\right)=p$, then $e_{i j} \equiv p$.

(vi) The image of $e_{i j}$ is contained in

$$
X_{\alpha(i, j)} \cap X_{\alpha(i+1, j)} \cap X_{\alpha(i, j+1)} \cap X_{\alpha(i+1, j+1)} \text {. }
$$

(Assume $X_{\alpha(i, j)}=X$ if $i=0, n+1$ or if $j=0, m+1$ ).

(vii) If $\sum_{k=1}^{j-1}\left\|a_{k}\right\| \leq t_{i-1} \leq t_{i} \leq \sum_{k=1}^{j}\left\|a_{k}\right\|$, then the image of $e_{i 1}$ contained in $X_{\alpha}$.

Next, ef. Fig. 1, consider paths

$$
\begin{array}{ll}
c_{i j}(t)=h\left(t+t_{i-1}, s_{j}\right) & 0 \leq t \leq t_{i}-t_{i-1} \\
d_{i j}(s)=h\left(t_{i}, s+s_{j-1}\right) & 0 \leq s \leq s_{j}-s_{j-1}
\end{array}
$$

and set

$$
\begin{array}{ll}
a_{i j}=e_{i-1, j} \cdot c_{i j} \cdot e_{i j}^{-1} & i=1, \cdots, n \text { and } j=0, \cdots, m \\
b_{i j}=e_{i, j-1} \cdot d_{i j} \cdot e_{i j}^{-1} & i=0, \cdots, n \text { and } j=1, \cdots, m
\end{array}
$$

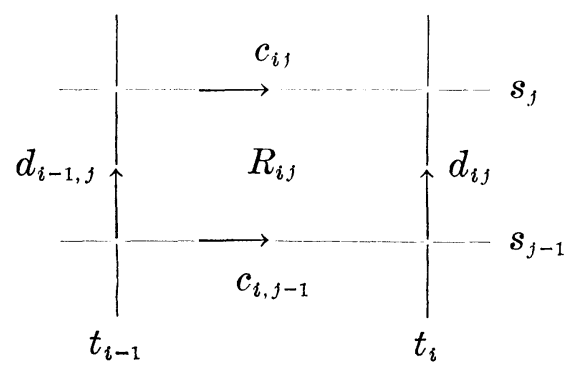

Fig. 1 
The image points of the loops $a_{i j}, b_{i j}, a_{i, j-1}$, and $b_{i-1, j}$ all lie in $X_{x(i, j)}$, Consequently, they define group elements $A_{i j}, B_{i j}, A_{i j}^{\prime}$, and $\beta_{i j}^{\prime}$ respectively. $G_{\alpha(i, j)}$. The product $a_{i, j-1} \cdot b_{i j} \cdot a_{i j}^{-1} \cdot b_{i-1, j}^{-1}$ is obviously contractible in $X$; moreover, since the image of $R_{i j}$ as well as the images of the four paths lies in $X_{\alpha(i, j)}$, the product is also contractible in $X_{x(i, j)}$. We may conclude that

$$
A_{i j}^{\prime} B_{i j} A_{i j}^{-1}\left(B^{\prime}{ }_{i j}\right)^{-1}=1 \text {. }
$$

The central idea in the proof of II is the fact that if group elements $A \in G_{\alpha}$ and $B \in G_{\beta}$ possess a common representative loop, then $\psi_{a} A=$ $\psi_{\beta} B$.

The proof is easy: By assumption the system $\mathfrak{S}$ contains the fundamental group $G_{\gamma}$ of the intersection $X_{\gamma}=X_{\alpha} \cap X_{\beta}$ and the homomorphisms

$$
G_{\alpha} \stackrel{\theta_{1}}{\longleftarrow} G_{\gamma} \stackrel{\theta_{2}}{\longrightarrow} G_{\beta}
$$

induced by inclusion. The assertion that $A$ and $B$ possess a common representative loop states that there exists a $p$-based loop $c$ in $X_{\gamma}$ such that $\theta_{1} c \in A$ and $\theta_{2} c \in B$. Thus, if $c$ defines $C \in G_{\gamma}$, we have

$$
\theta_{1} C=A \text { and } \theta_{2} C=B
$$

Since $\Psi$ is consistent with the mappings $\theta$,

$$
\psi_{\alpha} A=\psi_{\alpha} \theta_{1} C=\psi_{\gamma} C=\psi_{\beta} \theta_{2} C=\psi_{\beta} B .
$$

Applying the central assertion, we obtain

$$
\begin{aligned}
& \psi_{\alpha(i, j)} A_{i j}=\psi_{\alpha(i, j+1)} A_{i, j+1}^{\prime} \\
& \psi_{\alpha(i, j)} B_{i j}=\psi_{\alpha(i+1, j)} B_{i+1, j}^{\prime}
\end{aligned}
$$

Equation (1) says that the result of reading around each $R_{i j}$ under the homorphism $\zeta_{\alpha(i, j)}^{\prime}$ is the identity. Equations (2) show that edges of adjacent rectangles will cancel. It follows (by induction) that the result of reading around the circumference of the large rectangle $R$ is the identity. Furthermore, only the elements along the bottom edge, $s=0$, are non-trivial. We conclude, therefore, that

$$
\prod_{i=1}^{n} \psi_{\alpha(i, 0)} A_{i j}=1 \text {. }
$$

Since each of the numbers $\sum_{k=1}^{j}\left\|a_{k}\right\|, j=1, \cdots, r$, is a member of $\left\{t_{1}, \cdots, t_{n}\right\}$, there exists an index function $i(j)$ such that $i(0)=0$, and

$$
t_{i(j)}=\sum_{k=1}^{j}\left\|a_{k}\right\| \quad j=1, \cdots, r .
$$


Then,

$$
\prod_{i=i(j-1)+1}^{i(j)} a_{i 0} \simeq \varphi_{a_{j}} a_{j} \quad j=1, \cdots, r .
$$

However, by virtue of (vii), we may assume that the equivalence is in $X_{\alpha_{j}}$. Thus, each loop $a_{i 0}, i=i(j-1)+1, \cdots, i(j)$, determines a group element $A_{i}^{\prime} \in G_{\alpha_{j}}$ and

$$
\prod_{i=i(j-1)+1}^{i(j)} A_{i}^{\prime}=A_{j}
$$

Since $A_{i 0}$ and $A_{i}^{\prime}$ possess a common representative loop $a_{i 0}$, it follows from our central assertion that

$$
\psi_{a(i, 0)} A_{i j}=\psi_{a,} A_{i}^{\prime} \quad i=i(j-1)+1, \cdots, i(j),
$$

Finally, therefore,

$$
\begin{aligned}
1 & =\prod_{j=1}^{r} \prod_{i=i(j-1)+1}^{i(j)} \psi_{\alpha(i, 0)} A_{i 0}=\prod_{j=1}^{r} \prod_{i=i(j-1)+1}^{i(j)} \psi_{\alpha_{j}} A_{i}^{\prime} \\
& =\prod_{j=1}^{r} \psi_{\alpha_{j}} A_{j}
\end{aligned}
$$

and the proof of the generalized van Kampen theorem is complete.

4. Generators and relations. Since generators and relations describe a group only to within isomorphism, we shall speak of the image group of any direct limit of a system as the direct limit of the system. To obtain a presentation of the direct limit of a system of groups which are given by generators and relations is a simple matter of setting up the proper homomorphisms and chasing around a batch of consistent diagrams. Consider a system $\mathfrak{S}$, each group $G_{a}$ of which has a presentation (cf [3])

$$
G_{\alpha}=\left(x_{\alpha}^{1}, x_{\alpha}^{2}, \cdots: r_{\alpha}^{1}, r_{\alpha}^{2}, \cdots\right) .
$$

Each mapping $\theta: G_{\alpha} \rightarrow G_{\beta}$ in $\subseteq$ is described by giving the assignment $\theta x_{\alpha}^{i} \in G_{\beta}, i=1,2, \cdots$. Then, the direct limit of $\subseteq$ has the presentation

$$
\mathrm{G}=\left(\left\{x_{\alpha}^{i}\right\}:\left\{r_{\alpha}^{i}\right\},\left\{x_{\alpha}^{i}\left(\theta x_{\alpha}^{i}\right)^{-1}\right\}\right)
$$

i.e., all generators $x_{\alpha}^{i}$, all relators $r_{\alpha}^{i}$, and all elements $x_{\alpha}^{i}\left(\theta \alpha_{\alpha}^{i}\right)^{-1}$ (a proof is given in [4]). The presentation (1) can be simplified in that, for each homomorphism $\theta: G_{\alpha} \rightarrow G_{\beta}$, the relators $r_{\alpha}^{i}, i=1,2, \cdots$, may be dropped. The reason is that, in the free group $F$ generated by all the generators in (1) and of which $G$ is the homomorphic image, the relators $r_{\alpha}^{i}$ are a 
consequence of the relators $r_{\beta}^{i}$ and the elements $x_{\alpha}^{i}\left(\theta x_{\alpha}^{i}\right)^{-1}$. To prove this assertion consider the diagram

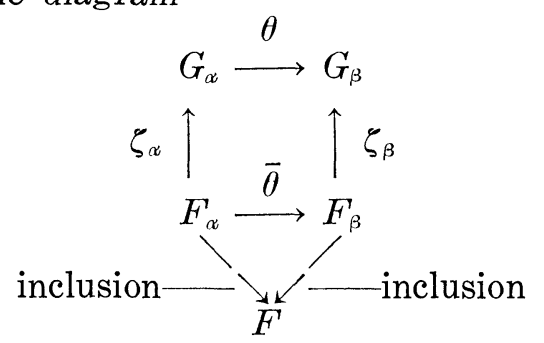

$F_{\alpha}$ is the free group generated by $x_{\alpha}^{i}, i=1,2, \cdots$, and $\zeta_{\alpha}$ is the cononical homomorphism whose kernel is the consequence of $r_{\alpha}^{i}, i=1,2, \cdots$. The mapping $\bar{\theta}$, which strictly speaking should be used in (1), is simply $\theta$ lifted to the free groups. Consider an arbitrary homomorphism $\eta$ of $F$ which maps $r_{\beta}^{i}, x_{\alpha}^{i}\left(\bar{\theta} x_{\alpha}^{i}\right)^{-1}, i=1,2, \cdots$, onto 1 . Then, for any $u \in F_{\alpha}$,

$$
\eta u=\eta \bar{\theta} u \text {. }
$$

Since

$$
\zeta_{\beta} \bar{\theta} r_{\alpha}^{i}=\theta \zeta_{\alpha} r_{\alpha}^{i}=1
$$

each $\bar{\theta} r_{\alpha}^{i}$ is a consequence of the elements $r_{\beta}^{i}$. Hence,

$$
\eta \bar{\theta} r_{\alpha}^{i}=\eta r_{\alpha}^{i}=1
$$

and the assertion is proved.

Consider a topogical space $X$ which is the union of two pathwiseconnected open subsets $X_{1}$ and $X_{2}$ whose intersection $X_{0}=X_{1} \cap X_{2}$ is also pathwise-connected and contains a point $p$. Suppose we are given presentations of the fundamental groups $G_{i}=\pi\left(X_{i}, p\right), i=0,1,2$,

$$
\begin{aligned}
G_{1} & =\left(x_{1}, x_{2}, \cdots: r_{1}, r_{2}, \cdots\right) \\
G_{2} & =\left(y_{1}, y_{2}, \cdots: s_{1}, s_{2}, \cdots\right) \\
G_{0} & =\left(z_{1}, z_{2}, \cdots: t_{1}, t_{2}, \cdots\right)
\end{aligned}
$$

and the mapping $\theta_{i}: G_{0} \rightarrow G_{i}, i=1,2$, induced by inclusion are described by assignments $\theta_{i} z_{j} \in G_{i}, i=1,2, j=1,2, \cdots$. By our principal Theorem (3.1) and the results of the preceding paragraph, the fundamental group $G=\pi(X, p)$ has the presentation

$$
G=\left(\left\{x_{j}\right\},\left\{y_{j}\right\},\left\{z_{j}\right\}:\left\{r_{j}\right\},\left\{s_{j}\right\},\left\{z_{j}\left(\theta_{i} z_{j}\right)^{-1}\right\}\right)
$$

This presentation is equivalent to (cf. [3])

$$
G=\left(\left\{x_{j}\right\},\left\{y_{j}\right\}:\left\{r_{j}\right\},\left\{s_{j}\right\},\left\{\theta_{1} z_{j}\left(\theta_{2} z_{j}\right)^{-1}\right\}\right)
$$


which is the assertion of the usnal formulation of the van Kampen theorem.

Consider a system $\mathfrak{S}$ of groups and mappings

$$
G_{1} \stackrel{\theta_{1}}{\longrightarrow} G_{2} \stackrel{\theta_{2}}{\longrightarrow} G_{3} \stackrel{\theta_{3}}{\longrightarrow} \ldots \text { with presentations }
$$

$$
G_{i}=\left(x_{i}^{1}, x_{i}^{2}, \cdots: r_{i}^{1}, r_{i}^{2}, \cdots\right)
$$

such that

$$
\theta_{i} x_{i}^{j}=x_{i+1}^{j} \text { and } \theta_{i} r_{i}^{j}=r_{i+1}^{j}
$$

$\left(G_{i+1}\right.$ may have more generators and relators than $\left.G_{i}\right)$. We may define a group

$$
G=\left(y_{1}, y_{2}, \cdots: s_{1}, s_{2}, \cdots\right)
$$

and a homomorphism $\Phi: \subseteq \rightarrow G$ such that

$$
\varphi_{i} x_{i}^{j}=y_{j} \text { and } \varphi_{i} r_{i}^{j}=s_{j} .
$$

It is easy to check that $G$ (more precisely, $\Phi: \subseteq \rightarrow G$ ) is the direct limit of $\mathfrak{S}$.

Finally, we consider an ascending chain of non-empty, open subsets $X_{1} \subset X_{2} \subset \cdots$ of some topological space. We have by (3.1) and (2.2) that the fundamental group $G$ of the union is the direct limit of the system

$$
G_{1} \stackrel{\theta_{1}}{\longrightarrow} G_{2} \stackrel{\theta_{2}}{\longrightarrow} \cdots,
$$

where $G_{i}=\pi\left(X_{i}, p\right)$ is the fundamental group and $\theta_{1}$ is induced by inclusion. Using the results of the preceding paragraph, we obtain a presentation (4) for $G$, if presentations (2) satisfying conditions (3), are given. This procedure is used in [1] to obtain (among other examples) a presentation of the group of the exterior of the Alexander Horned Sphere.

\section{REFERENCES}

1. W. A. Blankinship and R. H. Fox, Remarks on certain pathological open subsets of 3-space and their fundamental groups, Proc. Amer. Math. Soc., 1, (1950), 618-624.

2. R. H. Fox and E. Artin, Some wild cells and spheres in three-dimensional space, Ann. of Math., 49, (1948), 979-990.

3. R. H. Fox, Free differential calculus II, Ann. of Math. 59, (1954), 196-210.

4. R. H. Fox, Discrete groups and their presentations, Notes on lectures given spring term 1955 at Princeton University. 


\section{PACIFIC JOURNAL OF MATHEMATICS}

\section{EDITORS}

\section{David Gilbarg}

Stanford University

Stanford, California

R. A. Beaumont

University of Washington

Seattle 5 , Washington
A. L. Whiteman

University of Southern California

Los Angeles 7, California

L. J. PAIGE

University of California

Los Angeles 24, California

\section{ASSOCIATE EDITORS}
E. F. BECKENBACH
C. E. BURGESS
E. HEWITT
A. HORN

\author{
V. GANAPATHY IYER \\ R. D. JAMES \\ M. S. KNEBELMAN \\ L. NACHBIN
}
I. NIVEN
E. G. STRAUS
T. G. OSTROM
H. L. ROYDEN
G. SZEKERES
M. M. SCHIFFER
F. WOLF
K. YOSIDA

\section{SUPPORTING INSTITUTIONS}

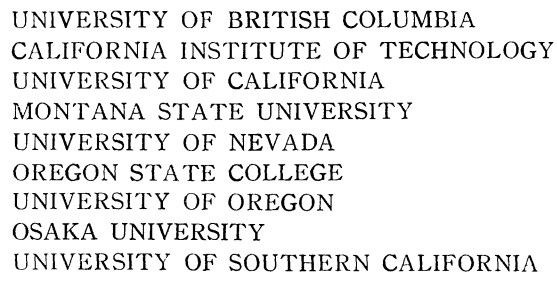

UNIVERSITY OF BRITISH COLUMBIA CALIFORNIA INSTITUTE OF TECHNOLOGY UNIVERSITY OF CALIFORNIA MONTANA STATE UNIVERSITY UNIVERSITY OF NEVADA OREGON STATE COLLEGE UNIVERSITY OF OREGON OSAKA UNIVERSITY UNIVERSITY OF SOUTHERN CALIFORNIA

STANFORD UNIVERSITY

UNIVERSITY OF TOKYO

UNIVERSITY OF UTAH

WASHINGTON STATE COLLEGE

UNIVERSITY OF WASHINGTON

AMERICAN MATHEMATICAL SOCIETY CALIFORNIA RESEARCH CORPORATION HUGHES AIRCRAFT COMPANY SPACE TECHNOLOGY LABORATORIES

Printed in Japan by Kokusai Bunken Insatsusha (International Academic Printing Co., Ltd.), Tokyo, Japan 


\section{Pacific Journal of Mathematics}

\section{Vol. 9, No. 1 \\ May, 1959}

Julius Rubin Blum and Murray Rosenblatt, On the structure of infinitely

divisible distributions . ............................. 1

Robert Geroge Buschman, Asymptotic expressions for

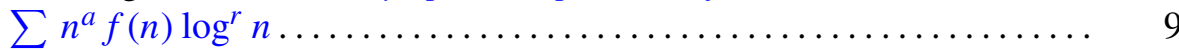

Eckford Cohen, A class of residue systems $(\bmod r)$ and related arithmetical

functions. I. A generalization of Möbius inversion .............. 13

Paul F. Conrad, Non-abelian ordered groups ................... 25

Richard Henry Crowell, On the van Kampen theorem............... 43

Irving Leonard Glicksberg, Convolution semigroups of measures ........ 51

Seymour Goldberg, Linear operators and their conjugates ............ 69

Olof Hanner, Mean play of sums of positional games .............. 81

Erhard Heinz, On one-to-one harmonic mappings ................ 101

John Rolfe Isbell, On finite-dimensional uniform spaces . . ........... 107

Erwin Kreyszig and John Todd, On the radius of univalence of the function

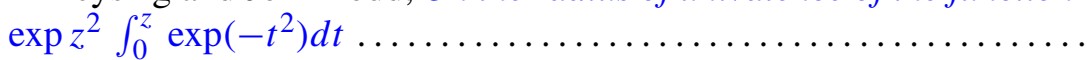

Roger Conant Lyndon, An interpolation theorem in the predicate

calculus......................................... 129

Roger Conant Lyndon, Properties preserved under homomorphism ........ 143

Roger Conant Lyndon, Properties preserved in subdirect products ....... 155

Robert Osserman, A lemma on analytic curves ................ 165

R. S. Phillips, On a theorem due to Sz.-Nagy..................... 169

Richard Scott Pierce, A generalization of atomic Boolean algebras ....... 175

J. B. Roberts, Analytic continuation of meromorphic functions in valued fields................................. 183

Walter Rudin, Idempotent measures on Abelian groups ................ 195

M. Schiffer, Fredholm eigen values of multiply-connected domains ........ 211

V. N. Singh, A note on the computation of Alder's polynomials ......... 271

Maurice Sion, On integration of 1-forms ...................... 277

Elbert A. Walker, Subdirect sums and infinite Abelian groups........... 287

John W. Woll, Homogeneous stochastic processes . .................. 293 